\title{
Debate: Goods, Games, and Institutions-Part 2
}

\section{“Goods, Games, and Institutions": A Reply}

\author{
Vinod K. AgGarwal and CÉdric Dupont
}

\begin{abstract}
In "Goods, Games, and Institutions," we discussed the underlying conditions under which institutions might promote cooperation among states. Our unified theoretical framework specifies bargaining games based both on the costs and benefits of different types of goods and actors' capabilities. Klaus Wallner argues in his critique in this issue that our modeling approach is seriously flawed. We show that Wallner comes to his conclusion because of several simple analytical errors on his part. After pointing out the flaws in his reasoning, we show that our framework is robust and can be easily extended to explore key features of international relations.
\end{abstract}

Keywords: • Bargaining games • International relations • Public goods provision

\section{Introduction}

In our article "Goods, Games, and Institutions," published in this journal (Aggarwal and Dupont, 1999), we emphasized the need to improve our understanding of the underlying conditions within which institutions might promote cooperation among states and other international actors. ${ }^{1}$ To do so, we provided a unified theoretical framework that specified bargaining games based both on the costs and benefits of different types of goods as well as the actors' differing capabilities. The attention received by that work suggests that our article has met one key objective-stimulating further work on formalizing the basis of international cooperation and the role of institutions.

Our emphasis in this comment is to examine whether our work will serve as a 
positive or negative benchmark for further research. According to Klaus Wallner, whose critique of our work appears on pages 343-401 in this issue, our modeling approach is seriously flawed. Consequently, while endorsing the thrust of our research program, he calls for a different analytical approach to examining the problems we identify. We will show here, however, that the perceived flaws identified by Wallner are really the result of several simple analytical errors on his part. And while his errors thus invalidate his own critique, his incorrect claims about the possibility of cost-sharing equilibria in the provision of public and common pool resource (CPR) goods have stimulated us to further develop our line of thinking.

Before turning to these findings on cost-sharing equilibria, however, we first discuss Wallner's errors. Of the four claims advanced by Wallner in his critique of our work, we focus on his central claim that we have made a serious analytical error in our modeling. All of his remaining claims derive from this assertion.

\section{Whose Error? An Invalid Critique}

The cornerstone of Wallner's criticism pertains to the structure of our game describing public good provision that we reproduce here as Table 1 (Table 2 in AD 1999). In AD Table 2, we showed how actors must choose whether or not to contribute to the creation of a public good or common pool resource.

Wallner claims that our strategy sets are "inadmissible" because "they violate the simultaneous-move assumption" of our normal form game. According to Wallner, players must choose a level of contribution, $c_{1}$ and $c_{2}$ under the constraint that $c_{1}+$ $c_{2}=c$, which presupposes that each player knows the other actor's level of contribution before he determines his own contribution. Wallner's flaw is that he misunderstands our strategy set: in our set-up, actors must contribute to the provision of a good by putting down resources equal to the cost of the good or by deciding not to contribute. The constraint $c_{1}+c_{2}=c$ applies to cases where both actors simultaneously decide to pay for the good. In such situations, players can get a partial refund when their commitment is matched by that of the other player (Aggarwal and Dupont, 1999: 397).

This is a very important assumption and one that Wallner ignores when he uses our payoff function to expand our $2 \times 2$ game to a $3 \times 3$ one. To see the error that he makes, consider Table 2.

According to our refund rule, when there are reciprocated commitments, the payoffs are $\left[b_{1}-c_{1}, b_{2}-c_{2}\right]$ with $c_{1}+c_{2}=c$. This implies that there is no difference between the outcomes (all, all), (all, half), (half, all) and (half, half). The payoff is $3(8-5)$ for each player. It appears that Wallner's payoff function is $\left[b_{1}-c, b_{2}-c\right]$.

TABLE 1. Payoff Structures for Public and CPR Goods (Payoffs of Player 1 First).

\begin{tabular}{l|c|c|}
\multirow{3}{*}{ Player 1/Player 2 } & \multicolumn{1}{c}{ Contribute } & Not contribute \\
\cline { 2 - 3 } Contribute & $\begin{array}{c}b_{1}-c_{1}, b_{2}-c_{2} \\
\text { or } \\
\left(-r_{1} \leq x_{1}<0\right),\left(-r_{2} \leq x_{2}<0\right)\end{array}$ & $\begin{array}{c}b_{1}-c, b_{2} \\
\text { or } \\
\left(-r_{1} \leq x_{1}<0\right), 0\end{array}$ \\
\cline { 2 - 3 } Not contribute & $\begin{array}{c}b_{1}, b_{2}-c \\
\text { or } \\
\left(-r_{2} \leq x_{2}<0\right)\end{array}$ & 0,0 \\
& &
\end{tabular}


TABLE 2. Correcting the False Correction for AD 1999 Table 4: Symmetric Resources with the Possibility of Unilateral Provision; $\left(b_{1}=b_{2}=8\right)$

Wallner's extension

\begin{tabular}{|c|c|c|}
\multicolumn{1}{c}{ All (10) } & Half (5) & None (0) \\
\hline$-2,-2$ & $-2,3$ & $-2,8$ \\
\hline $3,-2$ & 3,3 & $-5,0$ \\
\hline $8,-2$ & $0,-5$ & 0,0 \\
\hline
\end{tabular}

\begin{tabular}{c|c|c|c|}
\multicolumn{3}{c}{} & \multicolumn{3}{c}{ Correct extension } \\
\cline { 2 - 4 } All (10) & Half (5) & None (0) \\
\cline { 2 - 4 } All (10) & 3,3 & 3,3 & $-2,8$ \\
\cline { 2 - 4 } Half (5) & 3,3 & 3,3 & $-5,0$ \\
\cline { 2 - 4 } None (0) & $8,-2$ & $0,-5$ & 0,0 \\
\hline
\end{tabular}

Assumptions: a) fixed benefits $\left(b_{1}=b_{2}=8\right)$ for consuming the good;

b) cost of production, $c=10$;

c) share of costs of production (in case of all/all): $c_{1}=c_{2}=5$;

d) levels of individual resources: $r_{1}=r_{2}=12$.

Yet this would mean that in the case of (all, all), players would end up paying twice for the same good! Alternatively, he could receive his payoffs if he had a "no refund rule"-which seems very far-fetched-both for reciprocated and nonreciprocated commitments. The same logic for deriving payoffs applies to Tables 3 and 4. The purpose of this exercise is to show that Wallner's extension of our basic structure does not follow our assumptions. What does this imply for his findings?

Which equilibria of the $3 \times 3$ games are consistent with our basic assumptions? At first sight, one might be tempted to support Wallner's claim that there are many Nash cost-sharing equilibria in our games including the half-half outcome. Yet, in the corrected right-hand matrices in Tables 2, 3, and 4, one can readily see that the middle strategies are weakly dominated, and thus, one should not expect actors to play them. Indeed, any player might move away from the outcome and decide to contribute fully to the provision of the good given that both players are indifferent among any of the four upper left cells. But if any of the players starts to move, they will stop moving only if they reach the lower right cell in Table 2 , the lower left and upper right cells in Table 3, and the upper right cell in Table 4 . Indeed, these are the results that we predicted in our original framework.

TABLE 3. Correcting the False Correction for AD 1999 Table 4: Symmetric resources with the Possibility of Unilateral Provision; $\left(b_{1}=b_{2}=12\right)$

Wallner's extension

\begin{tabular}{|c|c|c|}
\multicolumn{1}{c}{ All (10) } & \multicolumn{1}{c}{ Half (5) } & None $(0)$ \\
\hline 2,2 & 2,7 & 2,12 \\
\hline 7,2 & 7,7 & $-5,0$ \\
\hline 12,2 & $0,-5$ & 0,0 \\
\hline
\end{tabular}

Correct extension

\begin{tabular}{c|c|c|c|}
\multicolumn{1}{c}{} & \multicolumn{1}{c}{ All (10) } & Half (5) & \multicolumn{1}{c}{ None (0) } \\
\cline { 2 - 4 } All (10) & 7,7 & 7,7 & 2,12 \\
\cline { 2 - 4 } Half (5) & 7,7 & 7,7 & $-5,0$ \\
\cline { 2 - 4 } None (0) & 12,2 & $0,-5$ & 0,0 \\
\cline { 2 - 4 } & &
\end{tabular}

Assumptions: a) fixed benefits $\left(b_{1}=b_{2}=12\right)$ for consuming the good;

b) cost of production, $c=10$;

c) share of costs of production (in case of all/all): $c_{1}=c_{2}=5$;

d) levels of individual resources: $r_{1}=r_{2}=12$. 
TABLE 4. Correcting the False Correction for AD 1999 Table 6: Asymmetric Resources with the Possibility of Unilateral Provision $\left(b_{1}=12, b_{2}=8\right)$

Wallner's extension

\begin{tabular}{|c|c|c|}
\multicolumn{1}{|c}{ All (10) } & Half (5) & None (0) \\
\hline $2,-2$ & 2,4 & 2,8 \\
\hline $6,-2$ & 6,4 & $-6,0$ \\
\hline $12,-2$ & $0,-4$ & 0,0 \\
\hline
\end{tabular}

Correct extension All (10) Half (5) None (0)

\begin{tabular}{|c|c|c|c|}
\hline & All (10) & Half (5) & None $(0)$ \\
\hline All (10) & 6,4 & 6,4 & 2,8 \\
\hline Part (6) & 6,4 & 6,4 & $-5,0$ \\
\hline None $(0)$ & $12,-2$ & $0,-5$ & 0,0 \\
\hline
\end{tabular}

None $(0)$

Assumptions: a) benefits proportional to levels of resources (2/3 of total resources);

b) cost of production, $c=10$;

c) share of costs of production (all/all): $c_{1}=6, c_{2}=4$ (1/3 of resources);

d) levels of individual resources: $r_{1}=18, r_{2}=12$.

In short, this discussion clearly shows that Wallner is excessively hasty in coming to his conclusions about equilibria. Indeed, if one strictly applies our payoff function to Wallner's 3x3 games, cost-sharing equilibria appear only if we abuse the Nash solution concept, something that Wallner himself warns us not to do.

\section{A Closer Look at Our Refund Assumption}

So far Wallner appears to be wrong in claiming that we have made an analytical error in our piece. As noted, our main findings are not altered in a 3x3 game setting. The more interesting issue, however, concerns the question of how different rules-or what we call refund charges-will affect payoffs and the resulting game equilibria. To address this issue, Wallner's formulation is inadequate. The following sequential construction as depicted in Figure 1 provides the correct framework in which to examine this question.

The first moves by players 1 and 2 are identical to the games that we developed in Tables 2-4. Each player chooses a level of investment $\left(i_{1}\right.$ and $\left.i_{2}\right)$ among three different options (full cost of the given good, half of the cost of the good, or nothing). But we now allow for a second move to describe the case where one

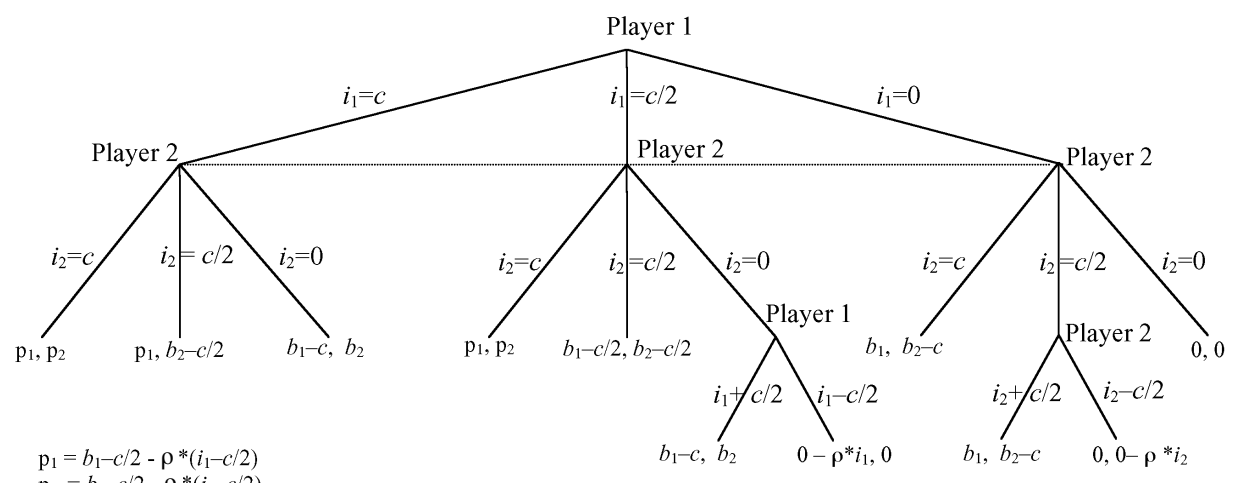

Figure 1. A Dynamic Structure of Interaction. 
player has invested less than the full cost of the good and the other player has not reciprocated. In these cases, the player who initially made an investment can either withdraw their investment or add to it in order to provide the good.

The idea behind permitting such a second move is to give the aggrieved actor an adjustment option. And it also allows us to evaluate how risky a choice of cost sharing might be for the players, depending on what we label "return charges." Return charges, a variable over the interval $[0,1]$ that we label $\rho$, refer to what a player can do if they find themselves with an unproductive investment that is either an under- $\left(i_{1}+i_{2}<c\right)$ or over-investment $\left(i_{1}+i_{2}>c\right)$. When greater than zero, return charges add a cost to any use of investment that is either insufficient to provide the good or in excess of the cost of providing the good.

Let us now consider a few specific numerical examples of this generic game, so that we can assess how, and whether, the outcomes of the structure of the new game differ from our original, more parsimonious framework. For the sake of presentation, we use the same numerical values as in Table 2 (symmetric resources with the possibility of unilateral provision). We use two extreme cases of return charges $(\rho=0$ and $\rho=1)$ and then generalize about the impact of return charges on the existence of cost-sharing equilibria.

First, consider the situation in which there is no return charge for over- or under-investments. There are two subgame-perfect Nash equilibria in the game depicted in Figure 2: ${ }^{2}(1)$ both players choose to contribute half of the cost $(5,5)$ initially; where there is underinvestment, the player who has put down an investment withdraws it; or (2) both players choose not to contribute in the first sequence of moves; where there is underinvestment, the player who has put down an investment withdraws it. ${ }^{3}$ Let us now consider the case of very high return charges $(\rho=1){ }^{4}$

The increase in return charges has a major effect on the game because it eliminates the cost-sharing equilibria at half, half $(5,5)$. To see this in Figure 3, consider player 1's initial decision to invest half the costs toward production of the good. Player 2's best response to such a decision now is not to reciprocate (as in Figure 2), but rather to invest nothing. Once player 1 sees that they are the only one who has invested resources for the production of the good, they have no incentive to withdraw their initial investment decision because of the high return

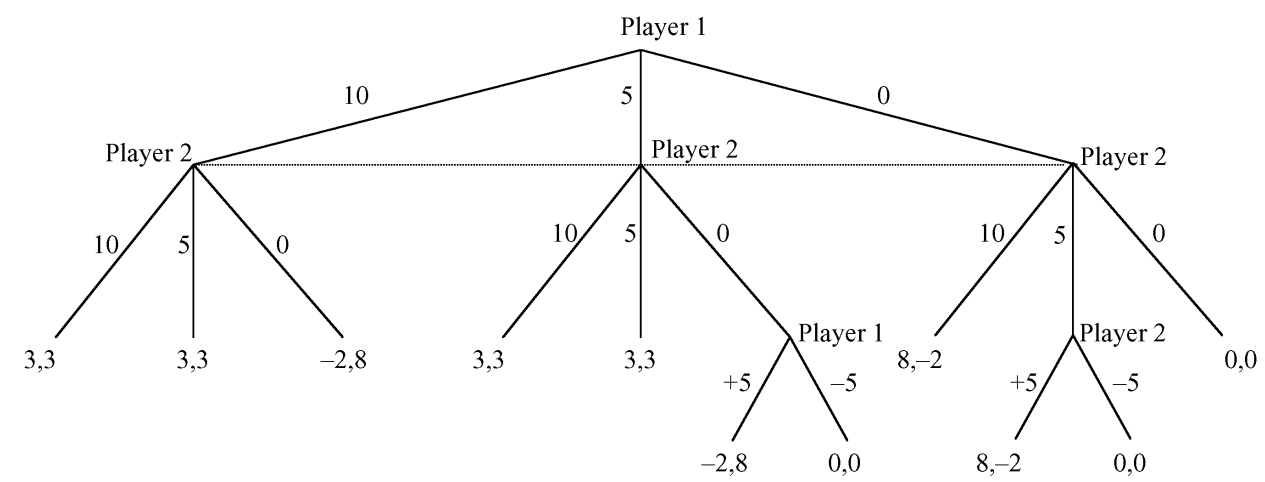

FIGURE 2. Symmetric Resources with Possibility of Unilateral Provision and No Return Charges $(\rho=0)$ 


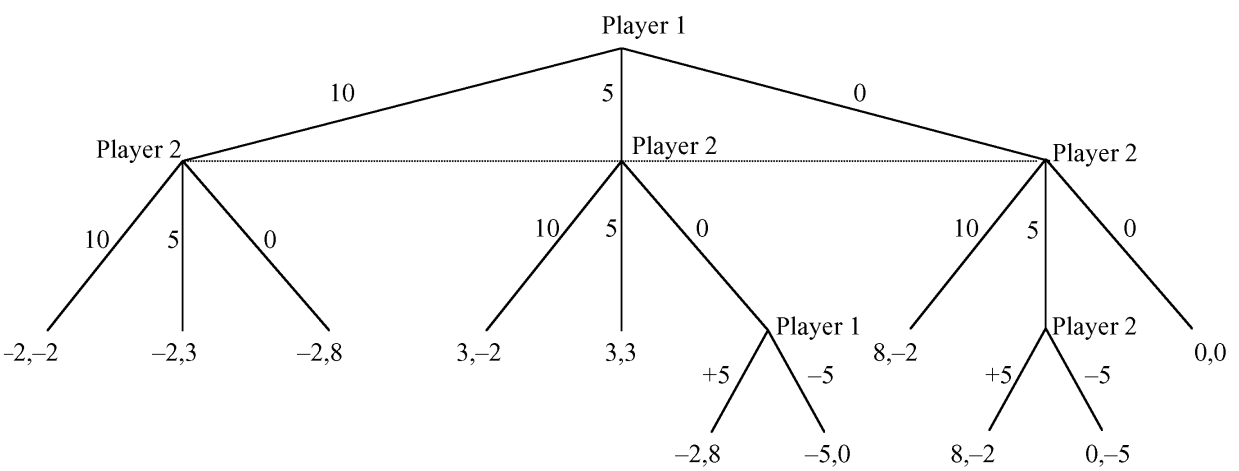

FIGURE 3. Symmetric Resources with Possibility of Unilateral Provision and High Return Charges

$$
(\rho=1) \text {. }
$$

changes. In this case, they will be motivated to invest their remaining resources (in the subgame starting after player 2's decision to invest zero, player 1 chooses +5 rather than -5). Similar reasoning applies to the case of player 2.

We can now generalize the relationship between return charges and the existence of cost-sharing equilibria. The latter depend critically upon the outcome of the subgame starting after limited but unreciprocated investments by any player. If there is an incentive for an aggrieved player to invest more, there is no cost-sharing equilibrium. With respect to the payoff structure presented in Figure 1 , this condition holds if $\left(b_{1}-c\right)>0-\rho * i_{1}$ and $\left(b_{2}-c\right)>0-\rho * i_{2}$, which can be rewritten as $\rho>\left(c-b_{1}\right) / i_{1}$ and $\rho>\left(c-b_{2}\right) / i_{2}$.

Using this double inequality, Figure 4 shows the range of $\rho$ values for which there are cost-sharing equilibria for interactions between players with symmetric large resources and medium benefits.

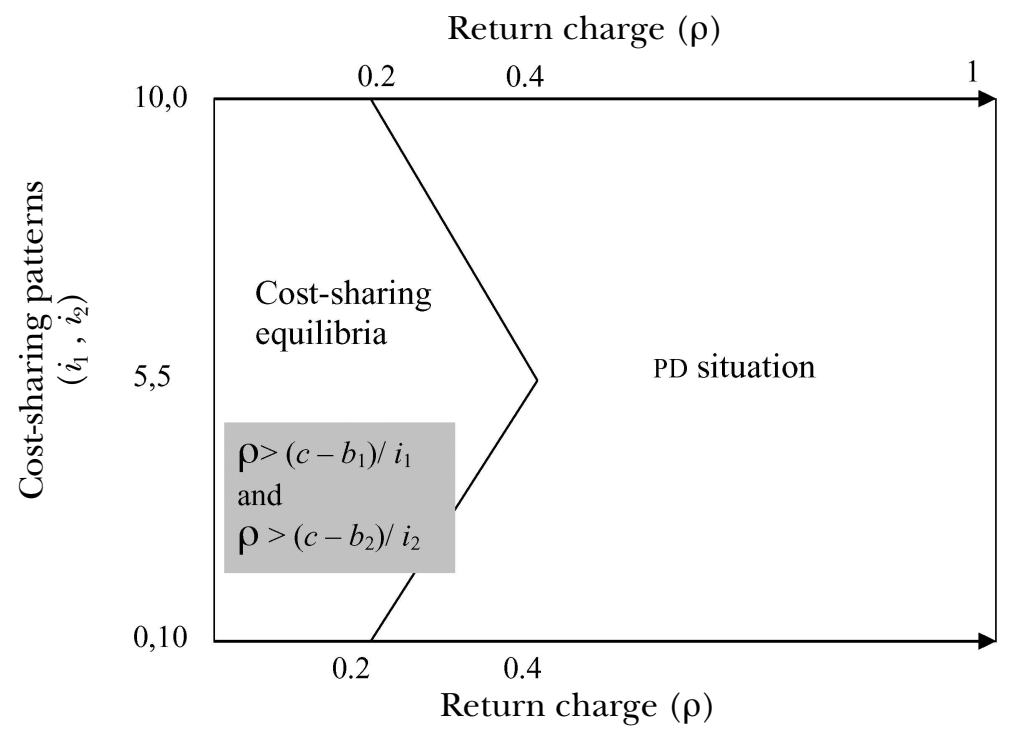

Figure 4. Range of Existence for Cost-sharing Equilibria for Basic Situation $b_{1}=b_{2}=8, c=10$. 
As can easily be seen in Figure 4, cost-sharing equilibria do not always exist, contrary to Wallner's claim, and their likelihood depends on the asymmetry of the sharing. The more asymmetric the distribution of costs between players, the lower the refund charges must be for the emergence of cost-sharing equilibria. Accordingly, cost-sharing equilibria are only a minor reason for a reduced emphasis on the prisoner's dilemma (PD) aspects of public and common pool resource goods in international relations. More interestingly, they matter only in PD situations. Applying our dynamic game structure to other types of strategic interaction mentioned in Wallner's critique, such as chicken (see Table 3 in this response), or mixed $\mathrm{PD} /$ chicken (see Table 4 in this response), we do not find any cost-sharing equilibrium, no matter what value $\rho$ takes. In these cases, the aggrieved actor always has an incentive to close the investment gap. Similar results emerge from an analysis of other game structures presented in AD (1999). The larger implication is that the claims we have developed in "Goods, Games, and Institutions" are robust to variations in return charges.

Let us consider the intuition underlying the concept of cost-sharing equilibria and return charges with a simple example. The classic public goods provision problem of constructing a lighthouse provides a ready entrée into these concepts. As is well known, a critical problem in the construction of public goods is the tendency by one or the other actor to free-ride, and thus the provision of such goods can be characterized as a prisoner's dilemma where each actor's dominant strategy is to defect.

What might cost sharing mean in such a situation? Simply put, if it takes 200 bricks to build the lighthouse, and if an actor loads 100 bricks into his car to meet his counterpart, then cost sharing would imply that both meet at the construction site and make their 100-brick contribution. But the public goods problem would not exist if each actor could be certain that the other would bring 100 bricks to the construction site. If we assumed that there were no costs involved in returning the bricks and driving back, then we could well expect that the actors might reach such a cost-sharing equilibrium. Alternatively, one could argue that any costsharing equilibrium would be possible: I would simply drive to the site with one brick and place it there, and then not contribute another brick until my counterpart did so.

This strategy is thus akin to extending the shadow of the future by slicing up a one-shot play of the PD into an iterated game. ${ }^{5}$ In such games, the so-called "folk theorem" asserts that a host of equilibria, including cost-sharing ones, are possible when players are sufficiently patient. ${ }^{6}$ The problem with this notion of the provision of public goods is that there is an important opportunity cost for actors of not having the good (which will, of course, vary with the utility of the good). While slicing up the problem of providing a lighthouse by returning every day with one brick helps to reduce uncertainty of the other actor's actions, our ships may be crashing on the shoals! Thus, waiting for a lengthy period of time can obviously be problematical. ${ }^{7}$

\section{Conclusion}

The lesson of this debate with Klaus Wallner is that, contrary to his assertion, the "necessary features of a suitable model linking preferences and technology to public goods provision" have been defined in "Goods, Games, and Institutions." The framework that we have developed is not only robust with respect to 
important changes in basic assumptions but can also be easily extended to explore key features of international relations. In addition to exploring the issue of return charges, we have also in more recent work examined the problem of the provision of club and private goods, and have sorted out the implications of adding players, uncertainty, and additional moves (Aggarwal and Dupont, 2000). Our current work focuses on situations involving the production of multiple goods, and highlights different linkage strategies-both issue and institutional. We have also been working on the empirical application of our framework. Systematic case studies that build on the insights developed here should help us to work toward our goal of enhancing our understanding of the microfoundations of institutional formation and change.

\section{Notes}

1. Since the discussion involves a number of tables from our original article and Klaus Wallner's critique of our work in this issue, we use the following notation: Aggarwal and Dupont 1999, AD 1999; Wallner 2001, w 2001; and this article AD 2001.

2. Subgame-perfection restricts the set of Nash equilibria to the ones that constitute an equilibrium profile in every proper subgame of the game, that is, here both for the initial simultaneous subgame and for the subgame starting after an asymmetric underinvestment. For an excellent discussion of subgame perfection, see Fudenberg and Tirole (1991: 92-100).

3. In this game, the players' initial equilibrium actions are similar to the ones in Wallner's game in Table 2.

4. Reduced to a normal form, this is a game that roughly corresponds to the one proposed by Wallner.

5. See, for example, Oye (1985).

6. See Fudenberg and Tirole (1991:150-160) for a discussion of the folk theorem.

7. In his critique, Wallner refers to the work of Admati and Perry (1991) in which the players pay for the good in alternating installments. But as noted, the urgency of good provision and the lack of attention to return charges mean that this approach may not be a feasible solution. In short, cost-sharing equilibria will appear only under very specific conditions, that is, when players discount future payoffs.

\section{References}

Admati, A.R. and M. Perry (1991). "Joint Prospects without Commitment." Review of Economic Studies, 58: 259-276.

Aggarwal, V.K. and C. Dupont (1999). "Goods, Games, and Institutions." International Political Science Review, 20(4): 393-409.

Aggarwal, V.K. and C. Dupont (2000). Goods, Games and Cooperation. Berkeley: University of California Press.

Fudenberg, D. and J. Tirole (1991). Game Theory. Cambridge, MA: MIT Press.

Oye, K..A. (1985). "Explaining Cooperation Under Anarchy.” World Politics, 38(1): 1-24.

Wallner, K. (2002). "The Provision of Public Goods in International Relations: A Comment on 'Goods, Games, and Institutions'.” International Political Science Review, this issue.

\section{Biographical Notes}

Vinod Aggarwal is Professor in the Department of Political Science, Affiliated Professor of Business and Public Policy in the Haas School of Business, and 
Director of the Berkeley Asia Pacific Economic Cooperation Study Center (BASC) at the University of California at Berkeley. He is the founder and Editor-in-Chief of the journal Business and Politics. His research focuses on international relations, international political economy, and theories of institutional change. His recent books include Institutional Designs for a Complex World (ed.) and Winning in Asia, European Style (ed.). ADDRESS: BASC, 802 Barrows Hall \#1970, University of California, Berkeley, CA 94720-1970, USA [e-mail: vinod@socrates.berkeley.edu].

CÉDric Dupont is Associate Professor of Political Science at the Graduate Institute of International Studies in Geneva and Associate Editor of Business and Politics. He has written on the political economy of international institutions in trade and monetary relations, bargaining theory, international negotiations, regional integration processes in Europe, Asia, and the Pacific region, and on Swiss foreign policy. ADDRESs: Graduate Institute of International Studies, 11A Avenue de la Paix, 1202 Geneva, Switzerland [e-mail: dupont@hei.unige.ch]. 